\title{
Post-traumatic ectopic calcification in the muscles of athletes: a review
}

\author{
J B King
}

\section{Introduction}

Post-traumatic ectopic calcification in muscles is well recognised but not very common. It occurs mainly in the anterior thigh muscles. The second most common site is the front of the upper arm and it occurs at other sites such as the calf and sole of the foot. In theory it can occur in any traumatised muscle.

Because of its potential to cause pain and loss of function, it is necessary to recognise it quickly and initiate early treatment. It appears to be more common in intramuscular as opposed to intermuscular bleeds and is recognisable by local muscle tenderness and, in particular, loss of stretch in the affected muscle group.

The classical treatment in the acute phase is rest, ice, compression, and elevation (RICE) with the addition of indomethacin when symptoms and signs are severe. There should be no return to training until a full stretch of the affected muscle group has been achieved.

Often when myositis ossificans has actually occurred, there is remarkably little dysfunction. In those who do have symptoms, excision of the ectopic bone should be delayed until the margins of the bone are smooth, suggesting maturity of the process. At that point surgical excision may be undertaken with postoperative drainage and indomethacin cover.

For the purposes of this article, the term myositis ossificans is used to describe ectopic calcification within a muscle. It does not include calcification that takes place after an avulsion of muscle or tendon from bone. In these two cases there are clearly bone-forming cells within the injured area.

\section{Materials and methods}

Two databases were used to review the literature: Ortholine (Knowledge Finder, Aries System Corporation) and Medline. Keywords used were post-traumatic calcification, myositis ossificans, Pelligrini-Steida disease, ectopic calcification, indomethacin, biphosphonates and muscle injury. Ortholine (using fuzzy logic) generated the 100 most likely references under any search heading, with a scale of likely relevance. Medline by contrast produced 350 references on myositis ossificans and 2089 references under indomethacin. Summaries of the apparently most relevant papers were selected. With a few exceptions, references that were more than 15 years old were discarded. The impact factor of the journals was checked before full reprints were sought. It was felt that it was impractical to produce a citation index.

The original objective had been a metaanalysis of treatment modalities and outcomes. However, most of the studies used very small numbers with no clear entry criteria and no good outcome markers, so this also became impractical. This paper therefore simply reflects the literature available with respect to some aspects of the pathological process.

\section{Occurrence}

Illes et $a l^{1}$ in their paper of 1992 quote Fay (1914) who stated that the condition was described by Copping in 1740 and Freke in 1741. Despite this long history, there is very little evidence as to the frequency of the condition.

The earliest worthwhile account of occurrence was in 1973 by Jackson and Feagin, ${ }^{2}$ who reported on the outcome of thigh contusions in West Point cadets. They reported a muscle calcification incidence following a direct blow of about $20 \%$. A further West Point study was performed by Ryan et $a l^{\beta}$ in 1991. They reported an incidence of $9 \%$ of myositis ossificans after quadriceps contusions. This study involved 117 cadets over three years with a minimal six month follow up, which included physical and $x$ ray examination. Rothwell ${ }^{4}$ in 1982 prospectively reviewed 60 thigh contusions and found an incidence of myositis ossificans of $17 \%$. Neither of the databases used discloses any other paper with a significant number of patients and this seems to be the only information available on the risk of occurrence. It should be noted that the occurrence rate appears to be diminishing, which may reflect changes in management.

With respect to ectopic calcification at other sites, there is very little in the literature other than the Jackson and Feagin ${ }^{2}$ and Ryan $^{3}$ papers, which confirm that the main incidence is in the anterior thigh muscles and the second most common site is brachialis. Carlson and Klassen ${ }^{5}$ suggest quite a high incidence in the arm (31 out of 83), but these results are for American Footballers and their paper therefore again reflects the North American experience where high energy transfer upper limb contact is more common.

\section{Diagnosis}

The history is relevant. There is no dispute in the literature that this condition is caused by a direct blow. Suggestions have been made that the amount of energy transferred is important, ${ }^{6}$ but no attempts have been made to measure this.

There is remarkable agreement that the physical signs suggesting impending myositis ossificans are localised tenderness, localised swelling, and lack of stretch. The West Point studies suggest that a limitation of flexion to $120^{\circ}$ is a significant predictor. No other study 
comments on the precise range of motion. In the West Point study the position of the hip is not given, which lessens the power of the observation, as the amount of knee flexion is completely dependent on the position of the hip. From personal experience, I have found that the extent of loss of stretch is often underestimated because the hip is allowed to flex.

Various laboratory tests have been suggested. Arrington and Miller ${ }^{7}$ report that serum alkaline phosphatase levels are elevated as is the erythrocyte sedimentation rate. Young et $a l^{\beta}$ suggest that lowered alkaline phosphatase levels are useful in establishing whether the lesion is mature but quote a paper in which alkaline phosphatase is not, in fact, mentioned. In two major review articles, Arrington and Miller ${ }^{7}$ and Young et $a l^{8}$ quote the use of nuclear medicine bone scanning in the early diagnosis of this condition and as an indicator of when the bone is mature. Neither group quotes its own experience and the references they use are well out of date. No recent publications on technetium bone scan and myositis ossificans were found.

Magnetic resonance imaging (MRI) has a place in the acute phase to define the position of the muscle lesion, particularly whether it is intramuscular. Once the myositis ossificans process starts, the images are difficult to interpret. (I have explored the vastus intermedius muscle on the suspicion of a malignant change, based on an altered homogeneous MRI signal. Histological examination confirmed widespread early calcification!) This view is supported by the papers of De Smet et $a l^{9}$ and Shirkhoda $e t a l .{ }^{10}$ Steinbach $e t ~ a l^{11}$ offer a review of MRI for muscle injuries.

\section{Risk factors}

The risk factors are quite well illustrated in the paper by Ryan et $a l{ }^{3}$ who highlight the lack of knee flexion to less than $120^{\circ}$. As previously mentioned, they did not refer to the position of the hip. As their treatment protocol included placing the knee into flexion, it is reasonable to suspect that the actual angle would be much less if measured with the hip in maximum extension. The rectus femoris crosses two joints and both have to be at the limit of motion to test the extensibility of the muscle. That being said, there is no doubt that lack of muscle stretch is a most important clinical sign.

The second risk factor in their paper was an American football injury, which simply confirms the problems of a direct blow. In Europe it is more likely to be caused by an opponent's knee rather than a helmet.

Recurrent injury was the third risk factor; it is not made clear whether this was a genuine second injury or a premature return to activity.

A sympathetic knee effusion was noted in just over half of those who developed myositis ossificans and a third had treatment delayed for more than three days.

Lipscomb et $a l^{12}$ add aggressive inappropriate physiotherapy involving passive stretching beyond the pain-free range. Interestingly, this is not quoted in the later publications, perhaps because it has become so well recognised as a problem.

\section{Pathology}

Three different forms of myositis ossificans are usually described. ${ }^{78}$ The first is a rather flat new bone formation directly adjacent to the shaft of bone and the second is a mushroom like formation of new bone that is still attached to the bone. It is logical to assume that in both of these cases there has been a haematoma which has either been contained within the periosteum or has burst through it into the local soft tissue. It is obvious that plenty of bone-forming cells are readily available from the damage to the periosteum. These are probably the lesions most likely to cause problems in athletes simply because they affect both the muscle and bone and thus restrict excursion, although this view is only to some extent supported by Young et al. ${ }^{8}$ No paper could be found that specifically reports the relative disabilities caused by calcification free within the muscle compared with that attached to bone.

The intriguing question is what is the origin of the new bone forming within a traumatised muscle without damage to the adjacent periosteum? What is the stimulus and from where do the cells come? An elegant study by Hierton ${ }^{13}$ uses a rabbit model of myositis ossificans, which is basically limb immobilisation interrupted by episodes of brief forced movement. Such a model reliably produces ectopic bone formation in the vastus intermedius region, and the histological findings are similar to those in the human. A labelled microsphere technique was used to measure the local blood flow. The study suggests that there is a decrease in circulation to the traumatised area of muscle which eventually ossifies.

Illes et $a l^{1}$ point out that the disease is characterised by the progression of nondifferentiated rapidly proliferating connective tissue which forms mature bone. The origin of these bone-forming cells is not known and they used lectins to try to characterise them. Specimens of myositis ossificans were taken from eight different human sites and the cells were tested against three lectins to identify the glycoproteins. The specificity of the binding site suggested that the precursors of the boneforming cells were part of an undifferentiated cell population present in normal muscle. The morphological features suggested a bone marrow derivation rather than a myofibroblast origin.

This shows that the cells are not necessarily derived from the margins of the bone. The stimulus for their conversion to bone-forming cells is not known. This point was investigated in the bone healing literature, which suggests that a lowered oxygen tension may initiate the complex cascade that leads to bone formation.

\section{Treatment}

Those papers that mention early treatment ${ }^{2} 378$ all confirm the efficacy of using RICE. No opposing view was found in the literature. The only significant modification is that of the West 
Point study where the original resting position was in flexion. This was achievable only because the cadets had to be admitted to hospital so that they could avoid parade duties. It would not be feasible even in a professional soccer club to admit players with thigh contusions and rest them with both hip and knee at $90^{\circ}$ ! There seems to be general agreement that early mobilisation, particularly stretching, is likely to cause more damage. In the West Point study, rather early active movement was allowed in terms of the range of knee flexion but, as previously stated, this work was carried out with the hip flexed, which may give an overoptimistic view of the true quadriceps excursion.

A major issue in orthopaedics is ectopic calcification in the soft tissues surrounding the hip after total hip replacement. Many studies have suggested that indomethacin is effective in reducing the incidence of this complication. ${ }^{14}$ The presumption is that indomethacin, as a non-steroidal anti-inflammatory, inhibits prostaglandin synthesis and thus new bone formation. It seems to be accepted that indomethacin is more efficacious than other nonsteroidal anti-inflammatories, but it is not clear whether it has a specific action or whether it is simply a more powerful drug.

Blumentha $1^{15}$ in his review of 1987 discusses mechanisms of calcification inhibition. $\mathrm{He}$ makes the point that body fluids such as plasma are supersaturated with hydroxyapatite and he wonders why humans do not mineralise and turn to stone! Significant levels of magnesium and proteins in the plasma probably prevent this. This may be the background to a report in the Czech literature, ${ }^{16}$ only a summary of which can I access, suggesting magnesium supplements for prevention of myositis ossificans.

There are two potential mechanisms by which mineralisation can be initiated. The first is removal of an inhibitor, and the second is the presence of a nucleator that promotes hydroxyapatite crystal formation. In therapeutic terms, the most logical approach is to maintain inhibition, as there is no evidence that it is possible to alter the presence of nucleators that promote crystal formation. Some of the most important inhibitors contain P-O-P or P-C-P linkages. The former tend to be ATP or ADP. The latter are represented by the biphosphonates. There are no specific reports of the use of biphosphonates for post-traumatic myositis ossificans, but Devogelaar ${ }^{17}$ suggests that they are not contraindicated if the usual measures and indomethacin have failed. Patients should be told that it is an experimental procedure and be closely monitored and treated with second generation drugs.

\section{Indications for surgery}

There is general agreement that early surgery is contraindicated for this condition and indeed that surgery should only be undertaken on mature lesions. Various authors ${ }^{18-20}$ have suggested using plain $x$ ray films, ultrasound, or triple phase bone scans to assess when the bone formed is mature. Ackerman et $a l^{18}$ (plain films) and Kirkpatrick et $a l^{19}$ (ultrasound) present case reports. No good objective ways of assessing maturity of the ectopic bone were found in the literature.

\section{Discussion}

I was disconcerted to discover how little worthwhile literature there was on this subject, and indeed how difficult it was to access.

I have tried to exclude cases associated with head injury and renal and metabolic bone disease. There is no doubt that research in these fields has led to insights into some aspects of ectopic calcification resulting from sports injury, especially in the potential use of biphosphonates. Having said that, I do not believe that the mechanism of this type of ectopic calcification can be regarded as being the same as that in localised muscle after a traumatic episode.

I believe that the indications for surgery are a mechanical block of function which persists after the clinical signs of inflammation such as local tenderness, swelling, and restriction of motion have completely disappeared. It is my experience that the lesions that cause a mechanical block are those where the ectopic calcification is attached to the underlying bone, thus tethering the muscle, but I cannot find any views for or against this hypothesis in the literature.

Given that significant intramuscular trauma with local haemorrhage is implicated in the causation of this calcification, it seems logical to operate with meticulous haemostasis and drainage, and, as recommended in orthopaedic hip replacement surgery, to use indomethacin during the postoperative period.

A major problem is the lack of a good experimental model on which to test the potential use of biphosphonates or hyperbaric oxygen. I hope that further research in this field will be undertaken. There is also the need for a good prospective study in British contact sports such as rugby and football. There is clearly scope for further research into the origin of the bone-forming cells and more particularly into the stimulus for such bone formation.

There is no confirmation in the literature that use of indomethacin is effective in myositis ossificans, even though there is abundant evidence that it is effective in other situations involving ectopic calcification. Therefore a prospective trial is needed, and the numbers required suggest that it should be multicentred. It could also investigate the use of biphosphonates.

\section{Conclusion}

Post-traumatic ectopic calcification is an entity that can significantly interfere with sporting performance. A careful review of the literature suggests that the demographics of the condition are not adequately known but that there may be a reduction in incidence with earlier and more appropriate treatment. The diagnosis is basically clinical. Further research into early diagnosis, causation at the cellular level, and optimum treatment is essential. 
1 Illes T, Dubousset J, Szendröl M, et al. Characterisation of bone forming cells in post traumatic myositis ossificans by lectins. Pathol Res Pract 1992;188:172-6.

2 Jackson DW, Feagin JA. Quadriceps contusion in young athletes. F Bone foint Surg [Am] 1973;55:95-105.

3 Ryan JB, Wheeler JH, Hopkinson WJ, et al. Quadriceps contusions. Am f Sports Med 1991;19;3:299-304

Rothwell AG. Quadriceps haematoma. A prospective clinical study. Clin Orthop 1982;171:97-103.

5 Carlson WO, Klassen RA. Myositis ossificans of the upper extremity: a long term follow up. $\mathcal{F}$ Pediatr Orthop 1984;4:693-6.

6 Hughston JC, Whatley GS, Stone MM. Myositis ossificans traumatica. South Med F 1962;55:1167-70.

7 Arrington ED, Miller MD. Skeletal muscle injuries. Orthop Clin North Am 1995;26:411-22.

8 Young JL, Laskowski ER, Rock MG. Thigh injuries in athletes. Mayo Clin Proc 1993;68:1099-106.

9 De Smet AA, Norris MA, Fisher DR. Magnetic resonance imaging in myositis ossificans. Skeletal Radiol 1992;21:5037 .

10 Shirkhoda A, Armin AR, Bis KG, et al. MR imaging in myositis ossificans. F Magn Reson Imaging 1995;5:287-92.

1 Steinbach LS, Fleckenstein JL, Mink JH. Magnetic resonance imaging of muscle injuries. Orthopaedics 1994; 17:991-9.
12 Lipscomb AB, Thomas ED, Johnston RK. Treatment of myositis ossificans traumatica in athletes. Am $\mathcal{F}$ Sports Med 1976;4:111-20

13 Hierton C. Regional blood flow in experimental myositis ossificans. Acta Orthop Scand 1983;54:58-63.

14 Schmidt SA, Kjaersgaard-Andersen P, Pederson NW, et al. The use of indomethacin to prevent the formation of heterotopic bone after total joint replacement. 7 Bone foint Surg [Am] 1998;70:834-8.

15 Blumenthal NC. Mechanisms of inhibition of calcification. Clin Orthop 1989;247:279-89.

16 Ditmar R, Steidl L. The importance of magnesium in orthopaedics. Acta Chirurgiae Orthopaedicae et Traumatologiae Cechoslovaca (Praha) 1989;56:190-200.

17 Devogelaer JP. Clinical use of biphosphonates. Curr Opin Rheumatol 1996;8:384-91.

18 Ackerman L, Ramamurthy S, Jablokow V, et al. Case report 488. Skeletal Radiol 1988;17:310-14.

19 Kirkpatrick JS, Koman LA, Rovere GD. The role of ultrasound in the early diagnosis of myositis ossificans; a case report. Am F Sports Med 1987;15:179-81.

20 Mellerowicz H, Stelling E, Kefenbaum A. Diagnostic ultrasound in the athlete's locomotor system. Br F Sports Med 1990;24:31-9.

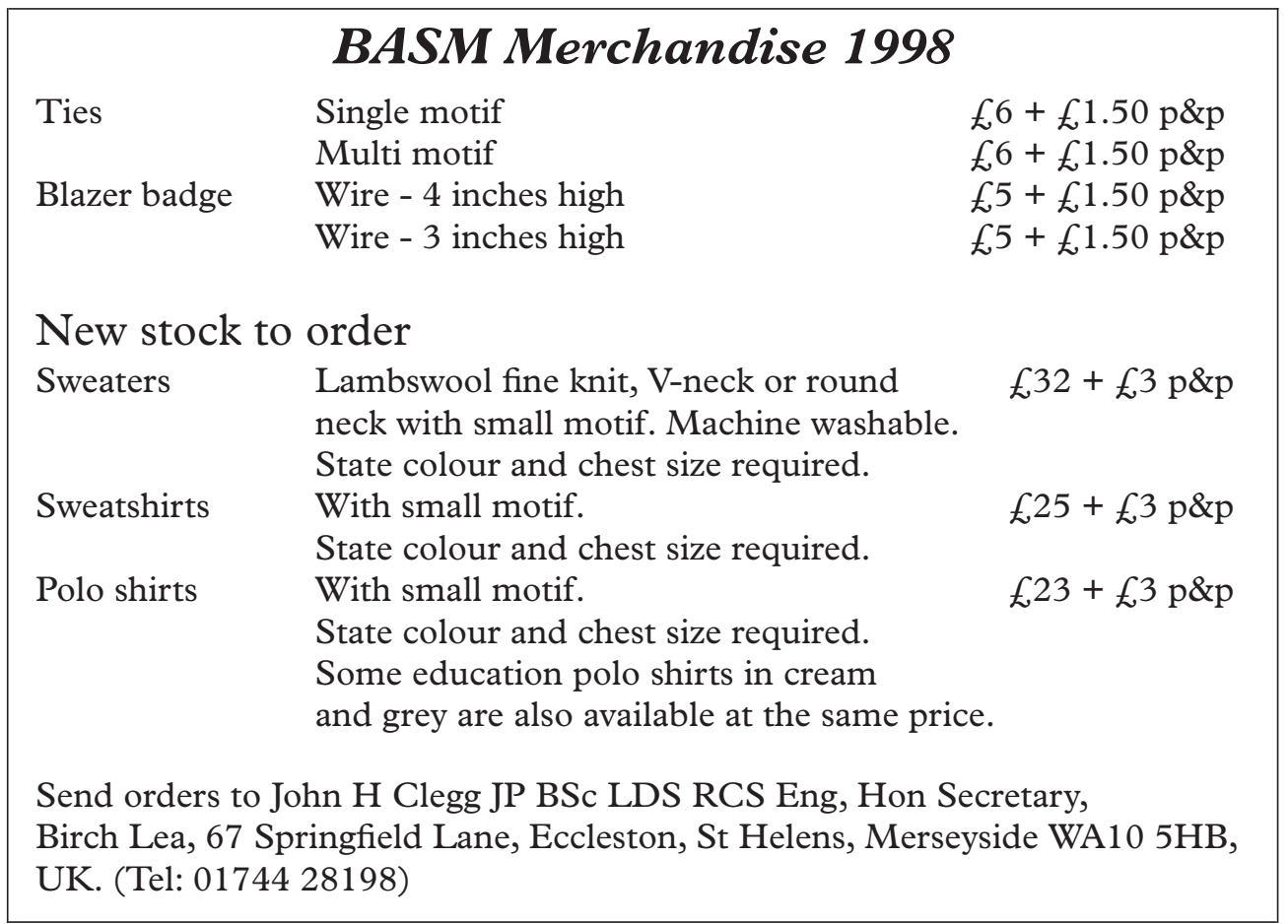

